\title{
Zonotopes with Large 2D-Cuts
}

\author{
Thilo Rörig • Nikolaus Witte • Günter M. Ziegler
}

Received: 26 October 2007 / Revised: 21 May 2008 / Accepted: 23 May 2008 /

Published online: 21 June 2008

(C) Springer Science+Business Media, LLC 2008

\begin{abstract}
There are $d$-dimensional zonotopes with $n$ zones for which a 2-dimensional central section has $\Omega\left(n^{d-1}\right)$ vertices. For $d=3$, this was known, with examples provided by the "Ukrainian easter eggs" by Eppstein et al. Our result is asymptotically optimal for all fixed $d \geq 2$.
\end{abstract}

Keywords Zonotopes · Cuts · Projections · Complexity · Ukrainian easter egg

\section{Introduction}

Zonotopes, the Minkowski sums of finitely many line segments, may also be defined as the images of cubes under affine maps, while their duals can be described as the central sections of cross polytopes. So, asking for images of zonotopes under projections or for central sections of their duals does not give anything new: We get again zonotopes resp. duals of zonotopes. The combinatorics of zonotopes and their duals is well understood (see, e.g., [18, Lect. 7]): The face lattice of a dual zonotope may be identified with that of a real hyperplane arrangement.

However, surprising effects arise as soon as one asks for sections of zonotopes resp. projections of their duals. Such questions arise in a variety of contexts.

The authors are supported by Deutsche Forschungsgemeinschaft, via the DFG Research Group "Polyhedral Surfaces," and a Leibniz grant.

T. Rörig · N. Witte · G.M. Ziegler $(\bowtie)$

MA 6-2, Inst. Mathematics, Technische Universität Berlin, 10623 Berlin, Germany

e-mail: ziegler@math.tu-berlin.de

T. Rörig

e-mail: thilosch@math.tu-berlin.de

N. Witte

e-mail: witte@math.tu-berlin.de 

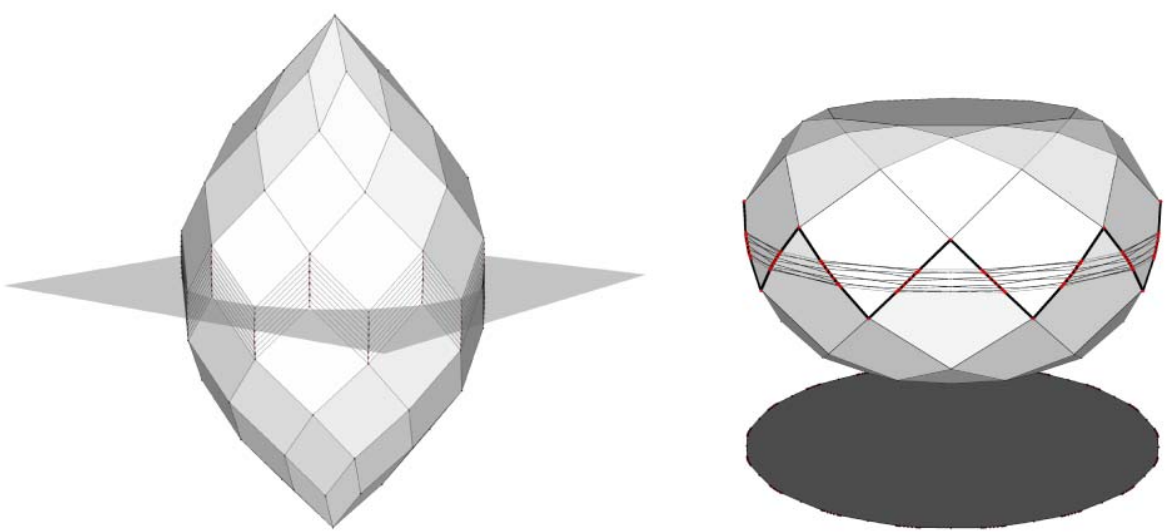

Fig. 1 Eppstein's Ukrainian easter egg and its dual. The 2D-cut, resp. shadow boundary, of size $\Omega\left(n^{2}\right)$ are marked

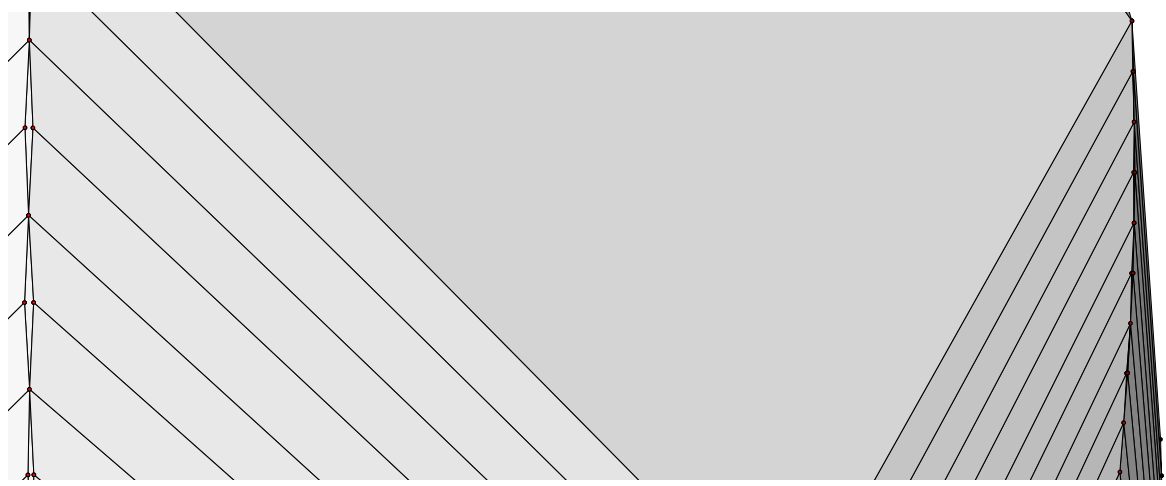

Fig. 2 Close-up view of an Ukrainian Easter egg

For example, the "Ukrainian Easter eggs" as displayed by Eppstein in his wonderful "Geometry Junkyard" [8] are 3-dimensional zonotopes with $n$ zones that have a 2dimensional section with $\Omega\left(n^{2}\right)$ vertices; see also Fig. 1. For "typical” 3-dimensional zonotopes with $n$ zones, one expects only a linear number of vertices in any section, so the Ukrainian Easter eggs are surprising objects. Moreover, such a zonotope has at most $2\left(\begin{array}{l}n \\ 2\end{array}\right)=O\left(n^{2}\right)$ faces, so any 2-dimensional section is a polygon with at most $O\left(n^{2}\right)$ edges/vertices, which shows that for dimension $d=3$, the quadratic behavior is optimal.

Eppstein's presentation of his model draws on work by Bern, Eppstein et al. [4], where also complexity questions are asked. (Let us note that it takes a closer look to interpret the picture given by Eppstein correctly: It is "clipped," and a close-up view shows that the vertical "chains of vertices" hide lines of diamonds; see Fig. 2.) Sections of zonotopes appear also in other areas such as Support Vector Machines and data depth; see [3, 7, 14]. (Thanks to Marshall Bern for these references.)

It is natural to ask for high-dimensional versions of the Easter eggs. 
Problem 1.1 What is the maximal number of vertices for a 2-dimensional central section of a d-dimensional zonotope with $n$ zones?

For $d=2$, the answer is trivially $2 n=\Theta(n)$, while for $d=3$, it is of order $\Theta\left(n^{2}\right)$, as seen above. We answer this question optimally for all fixed $d \geq 2$.

Theorem 1.2 For every $d \geq 2$, the maximal complexity (number of vertices) for a central $2 D$-cut of a d-dimensional zonotope $Z$ with $n$ zones is $\Theta\left(n^{d-1}\right)$.

The upper bound for this theorem is quite obvious: A $d$-dimensional zonotope with $n$ zones has at most $2\left(\begin{array}{c}n \\ d-1\end{array}\right)$ facets, thus any central 2D-section has at most $2\left(\begin{array}{c}n \\ d-1\end{array}\right)=$ $O\left(n^{d-1}\right)$ edges.

To obtain lower bound constructions, it is advisable to look at the dual version of the problem.

Problem 1.3 Koltun [17, Problem 3] What is the maximal number of vertices for a 2-dimensional affine image ( $a$ " $2 D$-shadow”) of a d-dimensional dual zonotope with $n$ zones?

Indeed, this question arose independently: It was posed by Vladlen Koltun based on the investigation of his "arrangement method" for linear programming (see [13]), which turned out to be equivalent to a Phase I procedure for the "usual" simplex algorithm (Hazan and Megiddo [12]). Our construction in Sect. 3 shows that the "shadow vertex" pivot rule is exponential in worst-case for the arrangement method.

Indeed, a quick approach to Problem 1.3 is to use known results about large projections of polytopes. Indeed, if $Z$ is a $d$-zonotope with $n$ zones, then the polar dual $Z^{*}$ of the zonotope $Z$ has the combinatorics of an arrangement of $n$ hyperplanes in $R^{d}$. The facets of $Z^{*}$ are $(d-1)$-dimensional polytopes with at most $n$ facetsand indeed every $(d-1)$-dimensional polytope with at most $n$ facets arises this way. It is known that such polytopes have exponentially large 2D-shadows, which in the old days was bad news for the "shadow vertex" version of the simplex algorithm $[11,15]$. Lifted to the dual $d$-zonotope $Z^{*}$, this also becomes relevant for Koltun's arrangements method; in Sect. 3 we briefly present this, and derive the $\Omega\left(n^{(d-1) / 2}\right)$ lower bound.

However, what we are really heading for is an optimal result, dual to Theorem 1.2. It will be proved in Sect. 4, the main part of this paper.

Theorem 1.2* For every $d \geq 2$, the maximal complexity (number of vertices) for a $2 D$-shadow of a d-dimensional zonotope $Z^{*}$ with $n$ zones is $\Theta\left(n^{d-1}\right)$.

\section{Basics}

Let $A \in \mathbb{R}^{m \times d}$ be a matrix. We assume that $A$ has full (column) rank $d$, that no row is a multiple of another one, and none is a multiple of the first unit-vector $(1,0, \ldots, 0)$. We refer to [5, Chap. 2] or [18, Lect. 7] for more detailed expositions of real hyperplane arrangements, the associated zonotopes, and their duals. 


\subsection{Hyperplane Arrangements}

The matrix $A$ determines an essential linear hyperplane arrangement $\widehat{\mathcal{A}}=\widehat{\mathcal{A}}_{A}$ in $\mathbb{R}^{d}$, whose $m$ hyperplanes are

$$
\widehat{H}_{j}=\left\{x \in \mathbb{R}^{d}: a_{j} x=0\right\} \quad \text { for } j=1, \ldots, m
$$

corresponding to the rows $a_{j}$ of $A$, and an affine hyperplane arrangement $\mathcal{A}=\mathcal{A}_{A}$ in $\mathbb{R}^{d-1}$, whose hyperplanes are

$$
H_{j}=\left\{x \in \mathbb{R}^{d-1}: a_{j}\left(\begin{array}{l}
1 \\
x
\end{array}\right)=0\right\} \quad \text { for } j=1, \ldots, m .
$$

Given $A$, we obtain $\mathcal{A}$ from $\widehat{\mathcal{A}}$ by intersection with the hyperplane $x_{0}=1$ in $\mathbb{R}^{d}$, a step known as dehomogenization; similarly, we obtain $\widehat{\mathcal{A}}$ from $\mathcal{A}$ by homogenization.

The points $x \in \mathbb{R}^{d}$ and hence the faces of $\widehat{\mathcal{A}}$ (and by intersection also the faces of $\mathcal{A}$ ) have a canonical encoding by sign vectors $\sigma(x) \in\{+1,0,-1\}^{m}$, via the map $s_{A}: x \mapsto\left(\operatorname{sign} a_{1} x, \ldots, \operatorname{sign} a_{m} x\right)$. In the following, we use the shorthand notation $\{+, 0,-\}$ for the set of signs. The sign vector system $s_{A}\left(\mathbb{R}^{d}\right) \subseteq\{+, 0,-\}^{m}$ generated this way is the oriented matroid [5] of $\widehat{\mathcal{A}}$.

The sign vectors $\sigma \in s_{A}\left(\mathbb{R}^{d}\right) \cap\{+,-\}^{m}$ in this system (i.e., without zeroes) correspond to the regions ( $d$-dimensional cells) of the arrangement $\widehat{\mathcal{A}}$. For a nonempty low-dimensional cell $F$, the sign vectors of the regions containing $F$ are precisely those sign vectors in $s_{A}\left(\mathbb{R}^{d}\right)$ which can be obtained from $\sigma(F)$ by replacing each " 0 " by either "+" or "-_".

\subsection{Zonotopes and Their Duals}

The matrix $A$ also yields the zonotope $Z=Z_{A}$ as

$$
Z=\left\{\sum_{i=1}^{m} \lambda_{i} a_{i}: \lambda_{i} \in[-1,+1] \text { for } i=1, \ldots, m\right\} .
$$

(In this set-up, $Z$ lives in the vector space $\left(\mathbb{R}^{d}\right)^{*}$ of row vectors, while the dual zonotope $Z^{*}$ considered below consists of column vectors.)

The dual zonotope $Z^{*}=Z_{A}^{*}$ may be described as

$$
Z^{*}=\left\{x \in \mathbb{R}^{d}: \sum_{i=1}^{m}\left|a_{i} x\right| \leq 1\right\} .
$$

The domains of linearity of the function $f_{A}: \mathbb{R}^{d} \rightarrow \mathbb{R}, x \mapsto \sum_{i=1}^{m}\left|a_{i} x\right|$, are the regions of the hyperplane arrangement $\widehat{\mathcal{A}}$. Their intersections yield the faces of $\widehat{\mathcal{A}}$, and these may be identified with the cones spanned by the proper faces of $Z^{*}$. Thus the proper faces of $Z^{*}$ (and, by duality, the nonempty faces of $Z$ ) are identified with sign vectors in $\{+, 0,-\}^{m}$ : These are the same sign vectors as we got for the arrangement $\widehat{\mathcal{A}}$.

Expanding the absolute values in (1) yields a system of $2^{m}$ inequalities describing $Z^{*}$. However, a nonredundant facet description of $Z^{*}$ can be obtained from $A$ 
and the combinatorics of $\widehat{\mathcal{A}}$ by considering the inequalities $\sigma(F) A x \leq 1$ for all sign vectors $\sigma(F)$ of maximal cells $F$ of $\widehat{\mathcal{A}}$ :

$$
Z^{*}=\left\{x \in \mathbb{R}^{d}: \sigma A x \leq 1 \text { for all } \sigma \in s_{A}\left(\mathbb{R}^{d}\right) \cap\{+,-\}^{m}\right\} .
$$

\subsection{Projections of Dual Zonotopes}

Let $P$ be a $d$-polytope, and let $F \subseteq P$ be a nonempty face. We define the matrix of normals $N_{F}$ as the matrix whose rows are the outer facet normals of all facets containing $F$. If $P=\left\{x \in \mathbb{R}^{d}: N x \leq b\right\}$ is given by an inequality description, then $N_{F}$ is the submatrix of $N$ formed by the rows of $N$ that correspond to inequalities that are tight at $F$. In the case where $P=Z^{*}$ is a dual zonotope, we derive the following description of $N_{F}$ that will be of great use later.

Lemma 2.1 Let $Z^{*}$ be a d-dimensional dual zonotope corresponding to the linear arrangement $\widehat{\mathcal{A}}$ given by the matrix $A$, and let $F \subset Z^{*}$ be a nonempty face. Then the rows of $N_{F}$ are the linear combinations $\sigma A$ of the rows of A for all sign vectors $\sigma \in s_{A}\left(\mathbb{R}^{d}\right)$ obtained from $\sigma(F)$ by replacing each " 0 ” by either "+" or "- ".

Let $F \subseteq P$ be a nonempty face of a $d$-polytope $P$, and consider a projection $\pi: \mathbb{R}^{d} \rightarrow \mathbb{R}^{k}$. If the outer normal vectors to the facets of $P$ that contain $F$, projected to the kernel of $\pi$, positively span this kernel, then $F$ is mapped to the face $\pi(F)$ of $\pi(P)$, which is equivalent to $F$, and $\pi^{-1}(\pi(F)) \cap P=F$. In this situation, we say that $F$ survives the projection.

Specialized to the projection $\pi_{k}: \mathbb{R}^{d} \rightarrow \mathbb{R}^{k}$ to the first $k$ coordinates and translated to matrix representations, this amounts to the following; see Fig. 3.

Lemma 2.2 (See, e.g., [16, 19]) Let $P$ be a d-polytope, $F$ a nonempty face, and let $N_{F}$ be its matrix of normals. If the rows of the matrix $N_{F}$, truncated to the last $d-k$ components, positively span $\mathbb{R}^{d-k}$, then $F$ survives the orthogonal projection $\pi_{k}$ to the first $k$ coordinates.

This "projection lemma" gives a sufficient condition for a face to survive. In a general position situation, when proper faces of $\pi(P)$ cannot be generated by higherdimensional faces of $P$, the condition of Lemma 2.2 is also necessary [16, Sect. 2.3].

Fig. 3 Survival of a face $F$ in the projection $\pi_{1}$ to the first coordinate

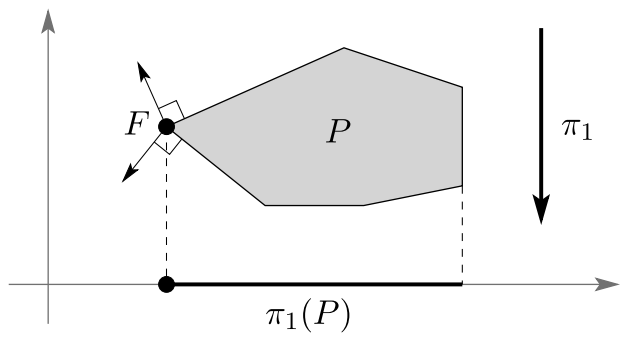




\section{Dual Zonotopes with Large 2D-Shadows}

In this section we present an exponential (yet not optimal) lower bound for the maximal size of 2D-shadows of dual zonotopes. It is merely a combination of known results about polytopes and their projections. For simplicity, we restrict to the case of odd dimension $d$.

Theorem 3.1 Let $d \geq 3$ be odd and $n$ an even multiple of $\frac{d-1}{2}$. Then there is a $d$ dimensional dual zonotope $Z^{*} \subset \mathbb{R}^{d}$ with $n$ zones and a projection $\pi: \mathbb{R}^{d} \rightarrow \mathbb{R}^{2}$ such that the image $\pi\left(Z^{*}\right)$ has at least $\left(\frac{2 n}{d-1}\right)^{\frac{d-1}{2}}$ vertices.

Here is a rough sketch of the construction.

(1) According to Amenta and Ziegler [2, Theorem 5.2] there are $(d-1)$-polytopes with $n$ facets and exponentially many vertices such that the projection $\pi_{2}: \mathbb{R}^{d-1} \rightarrow \mathbb{R}^{2}$ to the first two coordinates preserves all the vertices and thus yields a "large" polygon.

(2) We construct a $d$-dimensional dual zonotope $Z^{*}$ with $n$ zones that has such a $(d-1)$-polytope as a facet $F$.

(3) The extension of $\pi_{2}$ to the projection

$$
\pi_{3}: \mathbb{R} \times \mathbb{R}^{d-1} \rightarrow \mathbb{R}^{3},\left(x_{0}, x\right) \mapsto\left(x_{0}, \pi_{2}(x)\right),
$$

maps $Z^{*}$ to a centrally symmetric 3 -polytope $P$ with a large polygon as a facet. $P$ has a projection to $\mathbb{R}^{2}$ that preserves many vertices.

In the following, we give a few details to enhance this sketch.

Some details for (1): Here is the exact result by Amenta and Ziegler, which sums up previous constructions by Goldfarb [11] and Murty [15].

Theorem 3.2 (Amenta and Ziegler [2]) Let $d$ be odd and $n$ an even multiple of $\frac{d-1}{2}$. Then there is a $(d-1)$-polytope $F \subset \mathbb{R}^{d-1}$ with $n$ facets and $\left(\frac{2 n}{d-1}\right)^{\frac{d-1}{2}}$ vertices such that the projection $\pi_{2}: \mathbb{R}^{d-1} \rightarrow \mathbb{R}^{2}$ to the first two coordinates preserves all vertices of $F$. The polytope $F$ is combinatorially equivalent to a $\left(\frac{d-1}{2}\right)$-fold product of $\left(\frac{2 n}{d-1}\right)$ gons.

Explicit matrix descriptions of deformed products of $n$-gons with "large" 4dimensional projections are given in $[16,19]$. These can easily be adapted (indeed, simplified) to yield explicit coordinates for the polytopes of Theorem 3.2.

Some details for (2): We have to construct a dual zonotope $Z^{*}$ with $F$ as a facet.

Lemma 3.3 Given a $(d-1)$-polytope $F$ with $n$ facets, there is a d-dimensional dual zonotope $Z^{*}$ with $n$ zones that has a facet affinely equivalent to $F$. 
Proof Let $\left\{x \in \mathbb{R}^{d-1}: A x \leq b\right\}$ be an inequality description of $F$, and let $\left(-b_{i}, A_{i}\right)$ denote the $i$ th row of the matrix $(-b, A) \in \mathbb{R}^{n \times d}$.

The $n$ hyperplanes $H_{i}=\left\{x \in \mathbb{R}^{d}:\left(-b_{i}, A_{i}\right) x=0\right\}$ yield a linear arrangement of $n$ hyperplanes in $\mathbb{R}^{d}$, which may also be viewed as a fan (polyhedral complex of cones). According to [18, Cor. 7.18], the fan is polytopal, and the dual $Z^{*}$ of the zonotope $Z$ generated by the vectors $\left(-b_{i}, A_{i}\right)$ spans the fan.

The resulting dual zonotope $Z^{*}$ has a facet that is projectively equivalent to $F$; however, the construction does not yet yield a facet that is affinely equivalent to $F$. In order to get this, we construct $Z^{*}$ such that the hyperplane spanned by $F$ is $x_{0}=1$. This is equivalent to constructing $Z$ such that the vertex $v_{F}$ corresponding to $F$ is $e_{0}$. Therefore we have to normalize the inequality description of $F$ such that

$$
\sum_{i=1}^{n}\left(-b_{i}, A_{i}\right)=(1,0, \ldots, 0) \text {. }
$$

The row vectors of $A$ positively span $\mathbb{R}^{d-1}$ and are linearly dependent, hence there is a linear combination of the row vectors of $A$ with coefficients $\lambda_{i}>0, i=1, \ldots, n$, which sums to 0 . Thus if we multiply the $i$ th facet-defining inequality for $F$, corresponding to the row vector $\left(-b_{i}, A_{i}\right)$, by

$$
\frac{-\lambda_{i}}{\sum_{j=1}^{n} \lambda_{j} b_{j}},
$$

then we obtain the desired normalization of $A$ and $b$.

Some details for (3): The following simple lemma provides the last part of our proof; it is illustrated in Fig. 4.

Lemma 3.4 Let $P$ be a centrally symmetric 3-dimensional polytope, and let $G \subset P$ be a $k$-gon facet. Then there exists a projection $\pi_{G}: \mathbb{R}^{3} \rightarrow \mathbb{R}^{2}$ such that $\pi_{G}(P)$ is a polygon with at least $k$ vertices.
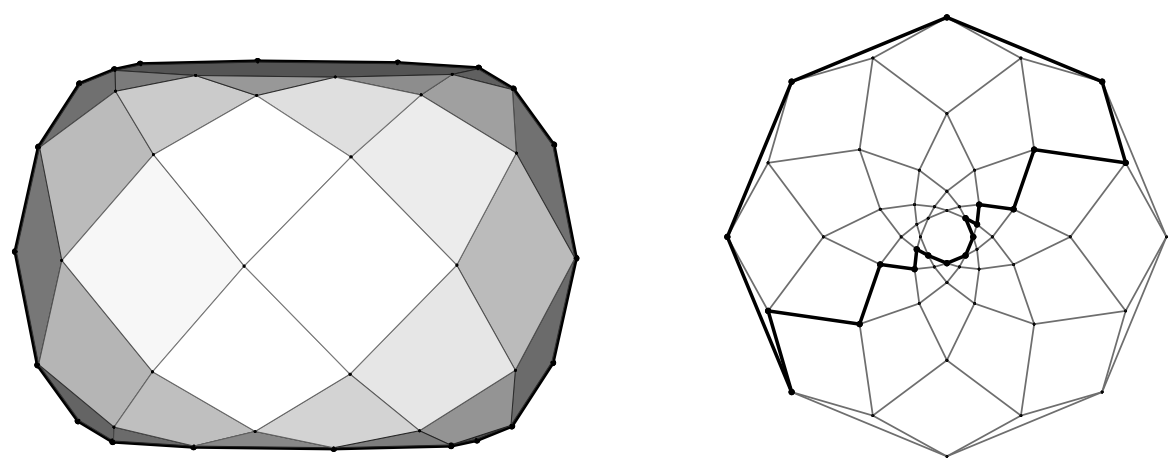

Fig. 4 Shadow boundary of a centrally symmetric 3-polytope, on the right displayed as its Schlegel diagram 
Proof Since $P$ is centrally symmetric, there exists a copy $G^{\prime}$ of $G$ as a facet of $P$ opposite and parallel to $G$. Consider a projection $\pi$ parallel to $G$ (and to $G^{\prime}$ ) but otherwise generic, and let $n_{G}$ be the normal vector of the plane defining $G$. If we perturb $\pi$ by adding $\pm \varepsilon n_{G}, \varepsilon>0$, to the projection direction of $\pi$, parts of $\partial G$ and $\partial G^{\prime}$ appear on the shadow boundary. Since $P$ is centrally symmetric, the parts of $\partial G$ and $\partial G^{\prime}$ appearing on the shadow boundary are the same. Therefore perturbing $\pi$ either by $+\varepsilon n_{G}$ or by $-\varepsilon n_{G}$ yields a projection $\pi_{G}$ such that $\pi_{G}(P)$ is a polygon with at least $k$ vertices.

\section{Dual Zonotopes with 2D-Shadows of Size $\Omega\left(n^{d-1}\right)$}

In this section we prove our main result, Theorem $1.2^{*}$, in the following version.

Theorem 4.1 For any $d \geq 2$, there is a d-dimensional dual zonotope $Z^{*}$ on $n(d-1)$ zones which has a $2 D$-shadow with $\Omega\left(n^{d-1}\right)$ vertices.

We define a dual zonotope $Z^{*}$ and examine its crucial properties. These are then summarized in Theorem 4.4, which in particular implies Theorem 4.1. Figure 5 displays a 3-dimensional example, and Fig. 8 a 4-dimensional example of our construction.

\subsection{Geometric Intuition}

Before starting with the formalism for the proof, which will be rather algebraic, here is a geometric intuition for an inductive construction of $Z^{*}=Z_{d}^{*} \subset \mathbb{R}^{d}$, a $d$ dimensional zonotope on $n(d-1)$ zones with a $2 \mathrm{D}$-shadow of size $\Omega\left(n^{d-1}\right)$ when
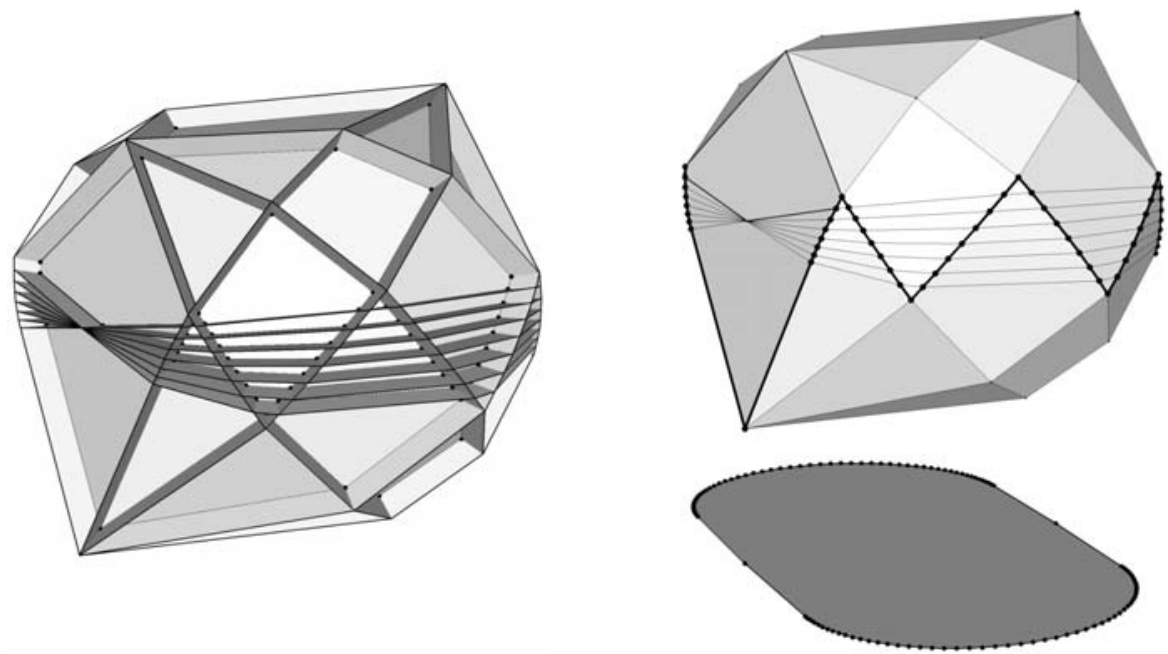

Fig. 5 A dual 3-zonotope with quadratic 2D-shadow, on the left with the corresponding linear arrangement and on the right with its $2 \mathrm{D}$-shadow 

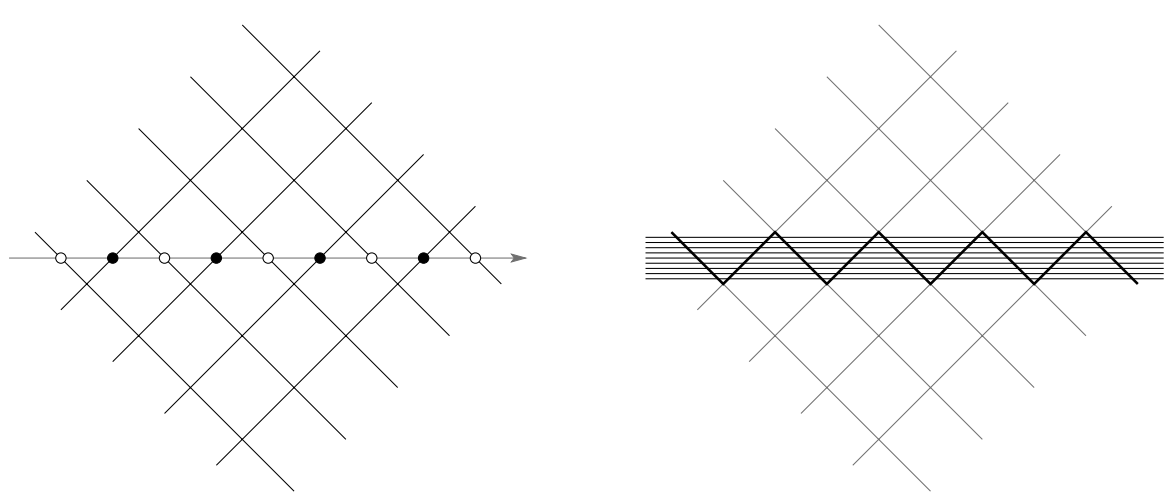

Fig. 6 Constructing the arrangement $\mathcal{A}_{3}^{\prime}$ from $\mathcal{A}_{2}$ (left) and $\mathcal{A}_{3}$ from $\mathcal{A}_{3}^{\prime}$ (right)

projected to the first two coordinates. For $d=2$, any centrally-symmetric $2 n$-gon (i.e., a 2-dimensional zonotope with $n$ zones) provides such a dual zonotope $Z_{2}^{*}$. The corresponding affine hyperplane arrangement $\mathcal{A}_{2} \subset \mathbb{R}^{1}$ consists of $n$ distinct points.

We derive a hyperplane arrangement $\mathcal{A}_{3}^{\prime} \subset \mathbb{R}^{2}$ from $\mathcal{A}_{2}$ by first considering $\mathcal{A}_{2} \times \mathbb{R}$ and then "tilting" the hyperplanes in $\mathcal{A}_{2} \times \mathbb{R}$. The hyperplanes in $\mathcal{A}_{2} \times \mathbb{R}$ are ordered with respect to their intersections with the $x_{1}$-axis. The hyperplanes in $\mathcal{A}_{2} \times \mathbb{R}$ are tilted alternatingly in $x_{2}$-direction as in Fig. 6 (left): Each black vertex of $\mathcal{A}_{2}$ corresponds to a north-east line and each white vertex becomes a northwest line of the arrangement $\mathcal{A}_{3}^{\prime}$. For each vertex in the $2 \mathrm{D}$-shadow of $Z_{2}^{*}$, we obtain an edge in the 2D-shadow of the dual 3-zonotope $Z_{3}^{* \prime}$ corresponding to $\mathcal{A}_{3}^{\prime}$. Now $\mathcal{A}_{3} \subset \mathbb{R}^{2}$ is constructed from $\mathcal{A}_{3}^{\prime}$ by adding a set of $n$ parallel hyperplanes to $\mathcal{A}_{3}^{\prime}$, all of them close to the $x_{1}$-axis, and each intersecting each edge of the 2D-shadow of $Z_{3}^{* \prime}$; see Fig. 6 (right).

For general $d$, let $\mathcal{H}_{d} \subset \mathcal{A}_{d}$ be the subarrangement of the $n$ parallel hyperplanes added to $\mathcal{A}_{d}^{\prime}$ in order to obtain $\mathcal{A}_{d}$. Then $\mathcal{A}_{d}^{\prime} \subset \mathbb{R}^{d-1}$ is constructed from $\mathcal{A}_{d-1} \times \mathbb{R}$ by tilting the hyperplanes $\mathcal{H}_{d-1} \times \mathbb{R}$, this time with respect to their intersections with the $x_{d-2}$-axis. The corresponding $d$-dimensional dual zonotope $Z_{d}^{* \prime}$ has $\Omega\left(n^{d-2}\right)$ edges in its $2 \mathrm{D}$-shadow and each of these $\Omega\left(n^{d-2}\right)$ edges is subdivided $n$ times by the hyperplanes in $\mathcal{H}_{d}$ when constructing $\mathcal{A}_{d}$, respectively $Z_{d}^{*}$. See Fig. 7 for an illustration of the arrangement $\mathcal{A}_{4}^{\prime}$.

\subsection{The Algebraic Construction}

For $k \geq 1, n=4 k+1$, and $d \geq 2$, we define

$$
\mathbf{b}=(k-i)_{0 \leq i \leq 2 k}=\left(\begin{array}{c}
k \\
\vdots \\
-k
\end{array}\right) \in \mathbb{R}^{2 k+1} \quad \text { and }
$$




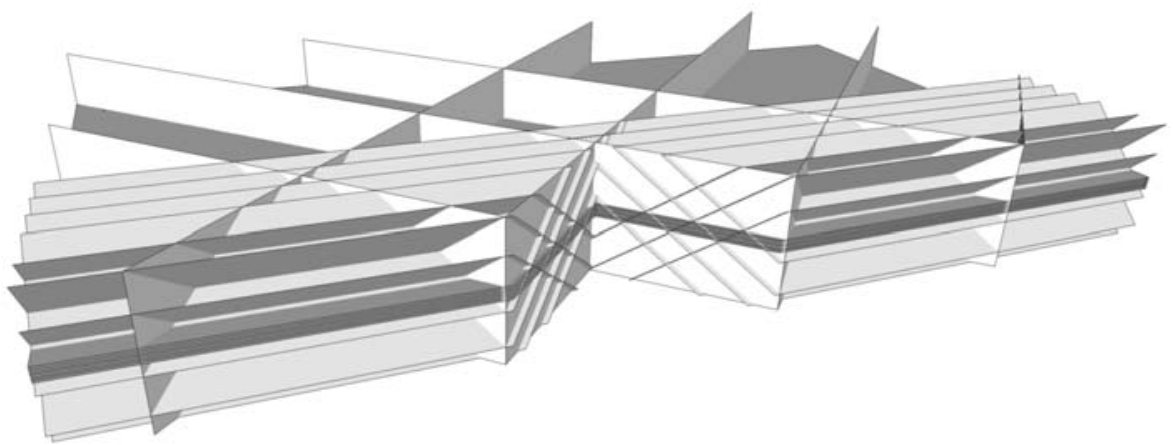

Fig. 7 The affine arrangement $\mathcal{A}_{4}$ consists of three families of planes: The first family $\mathcal{A}_{3}^{\prime} \times \mathbb{R}$ forms a coarse vertical grid; the second family (derived from $\mathcal{H}_{3} \times \mathbb{R}$ by tilting) forms a finer grid running from left to right; the last family $\mathcal{H}_{4}$ contains the parallel horizontal planes

$$
\mathbf{b}^{\prime}=\left(i-k+\frac{1}{2}\right)_{0 \leq i \leq 2 k-1}=\left(\begin{array}{c}
-k+\frac{1}{2} \\
\vdots \\
k-\frac{1}{2}
\end{array}\right) \in \mathbb{R}^{2 k}
$$

Let $\mathbb{O}, \mathbb{1} \in \mathbb{R}^{\ell}$ denote vectors with all entries equal to 0 , respectively 1 , of suitable size. For convenience, we index the columns of matrices from 0 to $d-1$ and the coordinates accordingly by $x_{0}, \ldots, x_{d-1}$. Let $\varepsilon_{i}>0$, and for $1 \leq i \leq d-1$, let $A_{i} \in$ $\mathbb{R}^{n \times d}$ be the matrix with $\varepsilon_{i}\left(\begin{array}{l}\mathbf{b} \\ \mathbf{b}^{\prime}\end{array}\right)$ as its 0th column vector, $\left(\begin{array}{c}\mathbb{1} \\ -\mathbb{1}\end{array}\right)$ as its $i$ th column vector, $\left(\begin{array}{l}\mathbb{1} \\ \mathbb{1}\end{array}\right)$ as its $(i+1)$ st column vector, and zeroes otherwise. In the case $i=d-1$, there is no $(i+1)$ st column of $A_{d}$ and the final $\left(\begin{array}{l}\mathbb{1} \\ \mathbb{1}\end{array}\right)$-column is omitted:

$$
A_{i}=\left(\begin{array}{ccccccccc}
0 & 1 & \cdots & i-1 & i & i+1 & i+2 & \cdots & d-1 \\
\varepsilon_{i} \mathbf{b} & \mathbb{0} & \cdots & \mathbb{0} & \mathbb{1} & \mathbb{1} & \mathbb{0} & \cdots & \mathbb{0} \\
\varepsilon_{i} \mathbf{b}^{\prime} & \mathbb{0} & \cdots & \mathbb{0} & -\mathbb{1} & \mathbb{1} & \mathbb{0} & \cdots & \mathbb{0}
\end{array}\right) \in \mathbb{R}^{(4 k+1) \times d}
$$

The linear arrangement $\widehat{\mathcal{A}}$ given by the $((d-1) n \times d)$-matrix $A$ whose horizontal blocks are the (scaled) matrices $\delta_{1} A_{1}, \ldots, \delta_{d-1} A_{d-1}$ for $\delta_{i}>0$ defines a dual zonotope by the construction of Sect. 2.2. Since the parameters $\delta_{i}$ do not change the arrangement $\widehat{\mathcal{A}}$, any choice of the $\delta_{i}$ yields the same combinatorial type of dual zonotope, but possibly different realizations. The choice of the $\varepsilon_{i}$ however may (and will for sufficiently large values) change the combinatorics of $\widehat{\mathcal{A}}$ and hence the combinatorics of the corresponding dual zonotope. For the purpose of constructing $Z^{*}$, we set $\alpha=\frac{1}{n+1}$ and $\varepsilon_{i}=\delta_{i}=\alpha^{i-1}$. This choice for $\varepsilon_{i}$ ensures that the "interesting" part of the next family of hyperplanes nicely fits into the previous family. Compare Fig. 6 (right): The interesting zig-zag part of family $A_{i}$ is contained by the interval $\varepsilon_{i}\left[-k-\frac{1}{4}, k+\frac{1}{4}\right]$ in the $x_{i}$-direction and by $\varepsilon_{i}\left[-\frac{1}{4}, \frac{1}{4}\right]$ in the $x_{i+1}$-direction; since $\varepsilon_{i+1}=\frac{1}{n+1} \varepsilon_{i}$, we obtain $\varepsilon_{i+1}\left(k+\frac{1}{4}\right)<\varepsilon_{i} \frac{1}{4}$, and the zig-zags nicely fit into each 
other. For these parameters, we obtain

$$
A=\left(\begin{array}{c}
A_{1} \\
\alpha A_{2} \\
\vdots \\
\alpha^{d-2} A_{d-1}
\end{array}\right)=\left(\begin{array}{cccc}
\mathbf{b} & \mathbb{1} & \mathbb{1} & \\
\mathbf{b}^{\prime}-\mathbb{1} & \mathbb{1} & \\
\alpha^{2} \mathbf{b}^{2} & \alpha \mathbb{1} & \alpha \mathbb{1} & \\
\alpha^{2} \mathbf{b}^{\prime} & -\alpha \mathbb{1} & \alpha \mathbb{1} & \\
\vdots & & \ddots & \\
\alpha^{2(d-2)} \mathbf{b} & & & \alpha^{d-2} \mathbb{1} \\
\alpha^{2(d-2)} \mathbf{b}^{\prime} & & & -\alpha^{d-2} \mathbb{1}
\end{array}\right) .
$$

This matrix has size $(d-1)(4 k+1) \times d=n(d-1) \times d$. The dual zonotope $Z^{*}=Z_{A}^{*}$ has $(d-1) n$ zones and is $d$-dimensional since $A$ has rank $d$. According to Sect. 2.1, any point $x \in \mathbb{R}^{d}$ is labeled in $\widehat{\mathcal{A}}$ by a sign vector $\sigma(x)=$ $\left(\sigma_{1}, \sigma_{1}{ }^{\prime} ; \sigma_{2}, \sigma_{2}{ }^{\prime} ; \ldots ; \sigma_{d-1}, \sigma_{d-1}{ }^{\prime}\right)$ with $\sigma_{i} \in\{+, 0,-\}^{2 k+1}$ and $\sigma_{i}{ }^{\prime} \in\{+, 0,-\}^{2 k}$. The following Lemma 4.2 selects $n^{d-1}$ vertices of $\mathcal{A}$.

Lemma 4.2 Let $H_{j_{1}}, H_{j_{2}}, \ldots, H_{j_{d-1}}$ be hyperplanes in $\mathcal{A}$, where each $H_{j_{i}}$ is given by some row $a_{j_{i}}$ of $A_{i}$, which is indexed by $j_{i} \in\{1, \ldots, n\}$. Then the $d-1$ hyperplanes $H_{j_{1}}, H_{j_{2}}, \ldots, H_{j_{d-1}}$ intersect in a vertex of $\mathcal{A}$ with sign vector $\left(\sigma_{1}, \sigma_{1}{ }^{\prime} ; \sigma_{2}, \sigma_{2}{ }^{\prime} ; \ldots ; \sigma_{d-1}, \sigma_{d-1^{\prime}}\right) \in\{+, 0,-\}^{n(d-1)}$ with 0 at position $j_{i}$ of the form

$$
\left(\sigma_{i}, \sigma_{i}{ }^{\prime}\right)= \begin{cases}(+\cdots+0-\cdots-,-\cdots-+\cdots+) & \text { with sum } 0 \text { or } \\ (+\cdots+-\cdots-,-\cdots-0+\cdots+) & \text { with sum } 0\end{cases}
$$

for each $i=1,2, \ldots, d-1$. Conversely, each of these sign vectors corresponds to $a$ vertex $v$ of the arrangement. In particular, $v$ is a generic vertex, i.e., $v$ lies on exactly $d-1$ hyperplanes.

Proof The intersection $v=H_{j_{1}} \cap H_{j_{2}} \cap \cdots \cap H_{j_{d-1}}$ is indeed a vertex since the matrix minor $\left(a_{j_{i}, \ell}\right)_{i, \ell=1, \ldots, d-1}$ has full rank. We solve the system $A^{\prime}\left(\begin{array}{l}1 \\ v\end{array}\right)=0$ to obtain $v$, where $A^{\prime}=\left(a_{j_{i}}\right)_{i=1, \ldots, d-1}$. As we will see, the entire sign vector of the vertex $v$ is determined by its " 0 " entries whose positions are given by the $j_{i}$. Hence every sign vector agreeing with (3) determines a set of hyperplanes $H_{j_{i}}$ and thus a vertex $v$ of the arrangement.

To compute the position of $v$ with respect to the other hyperplanes, we take a closer look at a block $A_{i}$ of the matrix that describes our arrangement. For an arbitrary point $x \in \mathbb{R}^{d}$ with $x_{0}=1$, we obtain

$$
A_{i} x=\left(\begin{array}{lrl}
\alpha^{i-1} \mathbf{b} & \mathbb{1} & \mathbb{1} \\
\alpha^{i-1} \mathbf{b}^{\prime} & -\mathbb{1} & \mathbb{1}
\end{array}\right)\left(\begin{array}{c}
1 \\
x_{i} \\
x_{i+1}
\end{array}\right)
$$

This is equivalent to the 2-dimensional(!) arrangement shown in Fig. 6 on the left. We will show that if $x$ lies on one of the hyperplanes and if $\left|x_{i+1}\right|<\frac{1}{4} \alpha^{i-1}$, then $x$ satisfies the required sign pattern (3). 
We start with an even simpler observation: If $x^{\prime}$ lies on one of the hyperplanes and has $x_{i+1}^{\prime}=0$ (so in effect we are looking at a 1-dimensional affine hyperplane arrangement), then there are:

$\triangleright 2 k$ "positive" row vectors $a_{j}$ of $A_{i}$ with $a_{j} x^{\prime}>0$,

$\triangleright 2 k$ "negative" row vectors $a_{j}$ of $A_{i}$ with $a_{j} x^{\prime}<0$, and

$\triangleright$ one "zero" row vector corresponding to the hyperplane $x^{\prime}$ lies on.

The order of the rows of $A_{i}$ is such that the signs match the sign pattern of $\left(\sigma_{i}, \sigma_{i}{ }^{\prime}\right)$ in (3). Since the values in $\alpha^{i-1} \mathbf{b}$ and $\alpha^{i-1} \mathbf{b}^{\prime}$ differ by at least $\frac{1}{2} \alpha^{i-1}$ we have in fact $a_{j} x^{\prime} \geq \frac{1}{2} \alpha^{i-1}$ for "positive" row vectors and $a_{j} x^{\prime} \leq-\frac{1}{2} \alpha^{i-1}$ for the "negative" row vectors of $A_{i}$. Hence we have

$$
\left|a_{j} x^{\prime}\right| \geq \frac{1}{2} \alpha^{i-1}
$$

If we now consider a point $x$ with $\left|x_{i+1}\right|<\frac{1}{4} \alpha^{i-1}$ on the same hyperplane as $x^{\prime}$, then $\left|x_{i}{ }^{\prime}-x_{i}\right|=\left|x_{i+1}\right|<\frac{1}{4} \alpha^{i-1}$. For the row vectors $a_{j}$ with $a_{j} x^{\prime} \neq 0$, we obtain:

$$
\begin{aligned}
\left|a_{j} x\right| & \geq\left|a_{j} x^{\prime}\right|-\left|a_{j}\left(x-x^{\prime}\right)\right| \geq \frac{1}{2} \alpha^{i-1}-\left(\left|x_{i}-x_{i}{ }^{\prime}\right|+\left|x_{i+1}-x_{i+1}{ }^{\prime}\right|\right) \\
& >\frac{1}{2} \alpha^{i-1}-\frac{1}{4} \alpha^{i-1}-\frac{1}{4} \alpha^{i-1}=0 .
\end{aligned}
$$

Hence the sign pattern of $x$ is the same as the sign pattern of $x^{\prime}$.

We conclude the proof by showing that the required upper bound $\left|v_{i+1}\right|<\frac{1}{4} \alpha^{i-1}$ holds for the coordinates of the selected vertex $v$. For all $i^{\prime}=1,2, \ldots, d-2$, the inequality $a_{j_{i^{\prime}}}\left(\begin{array}{l}1 \\ v\end{array}\right)=0$ directly yields the bound $\left|v_{i^{\prime}}\right| \leq k \alpha^{i^{\prime}-1}+\left|v_{i^{\prime}+1}\right|$. Further $a_{j_{d-1}}\left(\begin{array}{l}1 \\ v\end{array}\right)=0$ implies $\left|v_{d-1}\right| \leq k \alpha^{d-2}$ and thus recursively

$$
\begin{aligned}
\left|v_{i+1}\right| & \leq k \alpha^{i}+\left|v_{i+2}\right| \leq k \alpha^{i}+k \alpha^{i+1}+\left|v_{i+3}\right| \\
& \leq \cdots \leq k \alpha^{i}+k \alpha^{i+1}+\cdots+\left|v_{d-1}\right| \leq k \sum_{l=i}^{d-2} \alpha^{l}<k \alpha^{i} \sum_{l=0}^{\infty} \alpha^{l} \\
& =\frac{k \alpha^{i}}{1-\alpha}=\frac{k}{4 k+1} \alpha^{i-1}<\frac{1}{4} \alpha^{i-1} .
\end{aligned}
$$

The selected vertices of Lemma 4.2 correspond to certain vertices of the dual zonotope $Z^{*}$ associated to the arrangement $\mathcal{A}$. Rather than proving that these vertices of $Z^{*}$ survive the projection to the last two coordinates, we consider the edges corresponding to the sign vectors obtained from (3) by replacing the " 0 " in $\left(\sigma_{d-1}, \sigma_{d-1}^{\prime}\right)$ by either a "+" or a "-", and their negatives, which correspond to the antipodal edges.

Lemma 4.3 Let $S$ be the set of sign vectors $\pm\left(\sigma_{1}, \sigma_{1}{ }^{\prime} ; \sigma_{2}, \sigma_{2}{ }^{\prime} ; \ldots ; \sigma_{d-1}, \sigma_{d-1}{ }^{\prime}\right)$ of the form

$$
\left(\sigma_{i}, \sigma_{i}{ }^{\prime}\right)= \begin{cases}(+\cdots+0-\cdots-,-\cdots-+\cdots+) & \text { with sum } 0 \text { or } \\ (+\cdots+-\cdots-,-\cdots-0+\cdots+) & \text { with sum } 0\end{cases}
$$


for $1 \leq i \leq d-2$ and

$$
\left(\sigma_{d-1}, \sigma_{d-1}{ }^{\prime}\right)=(+\cdots+-\cdots-,-\cdots-+\cdots+) \quad \text { with sum } \pm 1 .
$$

Then the sign vectors in $S$ correspond to $2 n^{d-2}(n+1)$ edges of $Z^{*}$, all of which survive the projection to the first two coordinates.

Proof The sign vectors of $S$ indeed correspond to edges of $Z^{*}$ since they are obtained from sign vectors of nondegenerate(!) vertices by substituting one "0" by a " + " or a "-".

Further there are $2 n^{d-2}(n+1)$ edges of the specified type: Firstly, there are $n$ choices where to place the " 0 " in $\left(\sigma_{i}, \sigma_{i}{ }^{\prime}\right)$ for each $i=1, \ldots, d-2$, which accounts for the factor $n^{d-2}$. Let $p$ be the number of " + "-signs in $\sigma_{d-1}$. Thus there are $2 k+2$ choices for $p$, and for each choice of $p$, there are two choices for $\sigma_{d-1}{ }^{\prime}$, except for $p=0$ and $p=2 k+1$ with just one choice for $\sigma_{d-1}{ }^{\prime}$. This amounts to $2(2 k+2)-$ $2=n+1$ choices for $\left(\sigma_{d-1}, \sigma_{d-1}{ }^{\prime}\right)$. The factor of 2 is due to the central symmetry.

Let $e$ be an edge with sign vector $\sigma(e) \in S$. In order to apply Lemma 2.2 we need to determine the normals to the facets containing $e$. So, let $F$ be a facet containing $e$. The sign vector $\sigma(F)$ is obtained from $\sigma(e)$ by replacing each " 0 " in $\sigma(e)$ by either "+" or "-"; see Lemma 2.1. For brevity, we encode $F$ by a vector $\tau(F) \in\{+,-\}^{d-2}$ corresponding to the choices for " + " or "-" made. Conversely, there is a facet $F_{\tau}$ containing $e$ for each vector $\tau \in\{+,-\}^{d-2}$, since $e$ is nondegenerate.

The supporting hyperplane for $F$ is $a(F) x=1$ with $a(F)=\sigma(F) A$ being a linear combination of the rows of $A$. We compute the $i$ th component of $a(F)$ for $i=2,3, \ldots, d-1$ :

$$
\begin{aligned}
a(F)_{i} & =(\sigma(F) A)_{i}=\left(\left(\sigma_{i-1}, \sigma_{i-1}^{\prime}\right) A_{i-1}\right)_{i}+\left(\left(\sigma_{i}, \sigma_{i}^{\prime}\right) A_{i}\right)_{i} \\
& =\alpha^{i-2}\left(\sigma_{i-1}, \sigma_{i-1}^{\prime}\right)\left(\begin{array}{l}
\mathbb{1} \\
\mathbb{1}
\end{array}\right)+\alpha^{i-1}\left(\sigma_{i}, \sigma_{i}^{\prime}\right)\left(\begin{array}{r}
\mathbb{1} \\
-\mathbb{1}
\end{array}\right)
\end{aligned}
$$

Since we replace the zero of $\left(\sigma_{i-1}, \sigma_{i-1}^{\prime}\right)$ by $\tau(F)_{i-1}$ in order to obtain $\sigma(F)$ from $\sigma(e)$, we have $\left(\sigma_{i-1}, \sigma_{i-1}^{\prime}\right)\left(\begin{array}{l}\mathbb{1} \\ \mathbb{1}\end{array}\right)=\tau(F)_{i-1}$. Since $\left|\left(\sigma_{i}, \sigma_{i}^{\prime}\right)\left(\begin{array}{c}\mathbb{1} \\ -\mathbb{1}\end{array}\right)\right|$ is at most $n$, it follows that

$\triangleright a(F)_{i} \geq \alpha^{i-2}-n \alpha^{i-1}=\alpha^{i-1}>0$ holds for $\tau(F)_{i-1}=+$, and

$\triangleright a(F)_{i} \leq-\alpha^{i-2}+n \alpha^{i-1}=-\alpha^{i-1}<0$ holds for $\tau(F)_{i-1}=-$.

In other words, we have for $i=2,3, \ldots, d-1$ :

$$
\operatorname{sign} a(F)_{i}=\tau(F)_{i} .
$$

It remains to show that the last $d-2$ coordinates of the $2^{d-2}$ normals of the facets containing $e$, that is, the facets $F_{\tau}$ for all $\tau \in\{+,-\}^{d-2}$, span $\mathbb{R}^{d-2}$. But (4) implies that each of the orthants of $\mathbb{R}^{d-2}$ contains one of the (truncated) normal vectors $\left(a\left(F_{\tau}\right)_{i}\right)_{i=2, \ldots, d-1}$. Hence the (truncated) normals of all facets containing $e$ positively span $\mathbb{R}^{d-2}$, and $e$ survives the projection to the first two coordinates by Lemma 2.2. 

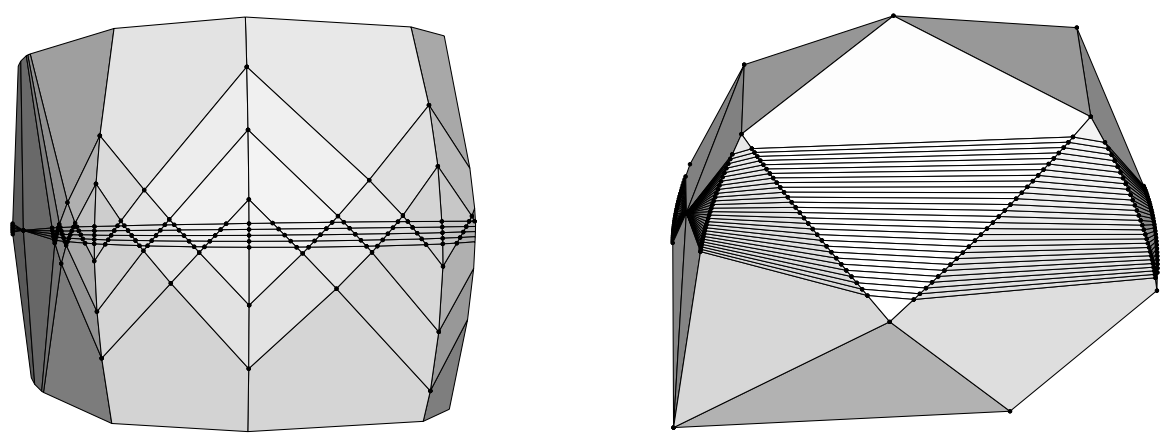

Fig. 8 Two different projections of a dual 4-zonotope with cubic 2D-shadow. On the left the projection to the first two and last coordinate (clipped in vertical direction) and on the right the projection to the first three coordinates

This completes the construction and analysis of $Z^{*}$. Scrutinizing the sign vectors of the edges specified in Lemma 4.3, one can further show that these edges actually form a closed polygon in $Z^{*}$. Thus this closed polygon is the shadow boundary of $Z^{*}$ (under projection to the first two coordinates), and its projection is a $2 n^{d-2}(n+1)$ gon. This yields the precise size of the projection of $Z^{*}$. The reader is invited to localize the edges corresponding to the closed polygon from Lemma 4.3 and the vertices from Lemma 4.2 in Figs. 6 and 7.

The following Theorem 4.4 summarizes the construction of $Z^{*}$ and its properties. Our main result as stated in Theorem 4.1 follows. Figure 5 displays a 3-dimensional example, and Fig. 8 a 4-dimensional example.

Theorem 4.4 Let $k$ and $d \geq 2$ be positive integers, and let $n=4 k+1$. The dual $d$ zonotope $Z^{*}=Z_{A}^{*}$ corresponding to the matrix A from (2) has $(d-1) n$ zones, and its projection to the first two coordinates has (at least) $2 n^{d-1}+2 n^{d-2}$ vertices.

Remark 4.5 As observed by Amenta and Ziegler [1, Sect. 5.2], any result about the complexity lower bound for projections to the plane (2D-shadows) also yields lower bounds for the projection to dimension $k$, a question which interpolates between the upper bound problems for polytopes/zonotopes $(k=d-1)$ and the complexity of parametric linear programming $(k=2)$, the task to compute the LP optima for all linear combinations of two objective functions (see [6, pp. 162-166]).

In this vein, from Theorem 4.1 and the fact that, in a dual of a cubical zonotope, every vertex lies in exactly $f_{k}\left(C_{d-1}\right)=\left(\begin{array}{c}d-1 \\ k\end{array}\right) 2^{k}$ different $k$-faces (for $k<d$ ) and that every such polytope contains at most $n^{d-1}$ faces of dimension $k$, one derives that, in the worst case, $\Theta\left(n^{d-1}\right)$ faces of dimension $k-1$ survive in a $k \mathrm{D}$-shadow of the dual of a $d$-zonotope with $n$ zones.

Acknowledgements We are grateful to Vladlen Koltun for his inspiration for this paper. Our investigations were greatly helped by use of the polymake system by Gawrilow and Joswig [9, 10]. In particular, we have built polymake models that were also used to produce the main figures in this paper. 


\section{References}

1. Amenta, N., Ziegler, G.M.: Shadows and slices of polytopes. In Proceedings of the 12th Annual ACM Symposium on Computational Geometry, May 1996, pp. 10-19

2. Amenta, N., Ziegler, G.M.: Deformed products and maximal shadows. In: Chazelle, B., Goodman, J.E., Pollack, R. (eds.) Advances in Discrete and Computational Geometry, South Hadley, MA, 1996. Contemporary Math., vol. 223, pp. 57-90. Am. Math. Soc., Providence (1998)

3. Bern, M.W., Eppstein, D.: Optimization over zonotopes and training support vector machines. In: Dehne, F.K.H.A., Sack, J.-R., Tamassia, R. (eds.) Proc. 7th Worksh. Algorithms and Data Structures (WADS 2001). Lecture Notes in Computer Science, vol. 2125, pp. 111-121. Springer, Berlin (2001)

4. Bern, M.W., Eppstein, D., Guibas, L.J., Hershberger, J.E., Suri, S., Wolter, J.D.: The centroid of points with approximate weights. In: Spirakis, P.G. (ed.) Proc. 3rd Eur. Symp. Algorithms, ESA 1995. Lecture Notes in Computer Science, vol. 979, pp. 460-472. Springer, Berlin (1995)

5. Björner, A., Las Vergnas, M., Sturmfels, B., White, N., Ziegler, G.M.: Oriented Matroids, 2nd edn., Encyclopedia of Mathematics, vol. 46. Cambridge University Press, Cambridge (1999)

6. Chvátal, V.: Linear Programming. Freeman, New York (1983)

7. Crisp, D.J., Burges, C.J.C.: A geometric interpretation of v-SVM classifiers. In: Solla, S.A., Leen, T.K., Müller, K.-R. (eds.) NIPS (Neural Information Processing Systems), vol. 12, pp. 244-250. MIT Press, Cambridge (1999)

8. Eppstein, D.: Ukrainian easter egg. In: "The Geometry Junkyard", computational and recreational geometry, 23 January 1997, http://www.ics.uci.edu/ eppstein/junkyard/ukraine/

9. Gawrilow, E., Joswig, M.: Polymake, version 2.3 (desert), 1997-2007, with contributions by Thilo Rörig and Nikolaus Witte, free software, http://www.math.tu-berlin.de/polymake

10. Gawrilow, E., Joswig, M.: Polymake: a framework for analyzing convex polytopes. In: PolytopesCombinatorics and Computation, Oberwolfach, 1997. DMV Seminars, vol. 29, pp. 43-73. Birkhäuser, Basel (2000)

11. Goldfarb, D.: On the complexity of the simplex algorithm. In: Advances in Optimization and Numerical Analysis. Proc. 6th Workshop on Optimization and Numerical Analysis, Oaxaca, Mexico, January 1992, pp. 25-38. Kluwer Academic, Dordrecht (1994). Based on: Worst case complexity of the shadow vertex simplex algorithm. Preprint, Columbia University 1983, 11 pages

12. Hazan, E., Megiddo, N.: The "arrangement method" for linear programming is equivalent to the phase-one method. IBM Research Report RJ10414 (A0708-017), IBM, August 292007

13. Koltun, V.: The Arrangement Method. Lecture at the Bay Area Discrete Math. Day XII, April 15, 2006, http://video.google.com/videoplay?docid=-6332244592098093013

14. Mosler, K.: Multivariate Dispersion, Central Regions and Depth. The Lift Zonoid Approach. Lecture Notes in Statistics, vol. 165. Springer, Berlin (2002)

15. Murty, K.G.: Computational complexity of parametric linear programming. Math. Program. 19, 213219 (1980)

16. Sanyal, R., Ziegler, G.M.: Construction and analysis of projected deformed products. Preprint, October 2007, 20 pages; http://arxiv.org/abs/0710.2162

17. Wagner, U.: Conference on geometric and topological combinatorics: problem session. Oberwolfach Rep. 4(1), 265-267 (2006)

18. Ziegler, G.M.: Lectures on Polytopes. Graduate Texts in Math., vol. 152. Springer, Berlin (1995), Revised 7th printing 2007

19. Ziegler, G.M.: Projected products of polygons. Electron. Res. Announc. Am. Math. Soc. 10, 122-134 (2004) 